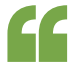

These

attributes

make them

attractive

alternatives for

implants and

actuators with

mechanical

properties

matching those

of living tissues
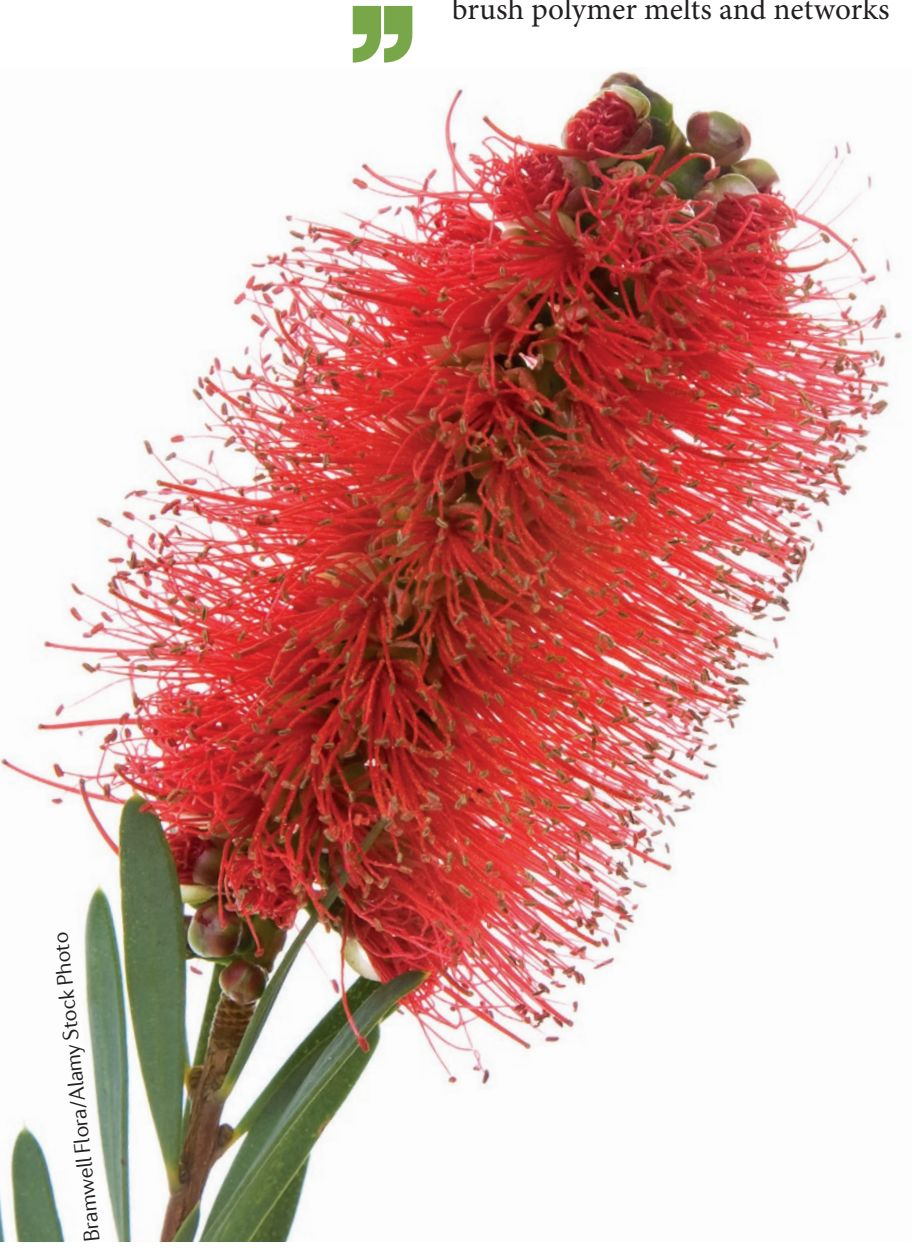

Entanglements within networks from telephone cables to polymer molecules - limit the mechanical properties of bulk materials. These physical interactions cause networks to stiffen and inhibit their extension. To disentangle molecules and, hence, create softer materials, a solvent is usually added; however, the introduction of a liquid fraction presents several practical problems, including phase separation and solvent leakage on deformation. Now, Sergei Sheiko and colleagues, at the University of North Carolina at Chapel Hill, have created bottlebrush polymer melts and networks

POLYMERS

\title{
Brushing off the solvent
}

that are superelastic and supersoft, even in the absence of a solvent.

The bottlebrush structures are synthesized, in a precise fashion, by grafting side chains to a polymer backbone using the atom transfer radical polymerization technique. This causes an increase in the diameter of individual polymer molecules, but, unlike typical filaments, the molecules remain quite flexible. As a result, there is a decrease in the entanglement density. Sheiko and colleagues predict the mechanical properties of polymers with systematically changing architectures - for example, with different degrees of polymerization of grafted chains and different grafting densities. Then, bottlebrush architectures comprising a variety of chemical building blocks are prepared and a universal structure-property correlation curve is established. "This architectural control of mechanical properties has reduced the limit of stiffness in dry polymer materials by 1,000 times, demonstrated uniaxial compressive deformability of up to nine times, and opened up new applications not available to stiffer materials or materials with liquid fractions," says Sheiko. "Furthermore, the bottlebrush polymers afford a multitude of chain ends that are amenable for various chemical modifications and enhance molecular freedom of motion, which directly affects numerous physical properties from glass transition and crystallization temperatures to enhancing their potential as adhesives."

The bottlebrush polymers show elasticity on a par with hydrogels. In addition, they overcome the problems of solvent evaporation and swelling that occur in polymer gels and, more specifically, hydrogels. However, it is in their potential biomedical applications in which the greatest advantages may lie. Polymer gels are frequently used to make soft and elastic biomedical implants because their mechanical properties are similar to those of living tissues; however, the solvent may elicit an inflammatory response in the surrounding tissue. The solvent-free bottlebrush polymers have a Young's modulus as low as $100 \mathrm{~Pa}$, which is approximately the same as that of brain tissue. These attributes make them attractive alternatives for implants and actuators with mechanical properties matching those of living tissues but avoiding the complications associated with solvent evaporation, leakage and tissue inflammation. "More specifically, these bottlebrush structures could be used alongside existing FDA-approved polymer materials to create stable and dry non-vascular biological implants for reconstructive surgery of fatty tissues that have been lost as a consequence of disease or trauma," adds Sheiko.

From a more fundamental perspective, the authors envisage exploring the molecular mechanisms of adhesion and toughness of the bottlebrush materials. "In addition, we will extend our studies to other types of branched architectures including comb-like polymers and explore the physical effect of the high number of chain ends with both inert and 'sticky' bristles," says Sheiko. With respect to possible applications, more diverse uses for the bottlebrush polymeric networks could be as highly extensible rubbers, soft pressure-sensitive adhesives, dielectric actuators, vibrational damping materials and cell-culture substrates.

Alison Stoddart

ORIGINAL ARTICLE Daniel, W. F. M. et al. Solvent-free, supersoft and superelastic bottlebrush melts and networks. Nat. Mater. http://dx.doi.org/10.1038/nmat4508 (2015) 